\title{
EXPONENTIAL ESTIMATES OF A CLASS OF TIME-DELAY NONLINEAR SYSTEMS WITH CONVEX REPRESENTATIONS
}

\author{
MÁximo RAMíREZ ${ }^{a}$, RAúl VillafuERTE $^{a, *}$, TemoATZin GONZÁLEZ $^{b}$, Miguel BERNAL $^{b}$ \\ ${ }^{a}$ Research Center on Information Technology and Systems \\ Hidalgo State University, Carretera Pachuca-Tulancingo Km. 4.5, CP 42184, Mineral de la Reforma, Hidalgo, Mexico \\ e-mail: villafuerte@uaeh.edu.mx \\ ${ }^{b}$ Department of Electric and Electronics Engineering \\ Sonora Institute of Technology, 5 de Febrero 818 Sur, CP 85000, Ciudad Obregón, Sonora, Mexico \\ e-mail: miguel.bernalaitson.edu.mx
}

\begin{abstract}
This work introduces a novel approach to stability and stabilization of nonlinear systems with delayed multivariable inputs; it provides exponential estimates as well as a guaranteed cost of the system solutions. The result is based on an exact convex representation of the nonlinear system which allows a Lyapunov-Krasovskii functional to be applied in order to obtain sufficient conditions in the form of linear matrix inequalities. These are efficiently solved via convex optimization techniques. A real-time implementation of the developed approach on the twin rotor MIMO system is included.
\end{abstract}

Keywords: exponential estimates, time delay systems, TS model, guaranteed cost, convex representations.

\section{Introduction}

In the framework of control theory it is well known that the existence of a delay in the control signal can induce instability or poor performance. It is clear that most of real systems present some sort of time delay, whether perceptible or not, besides being inherently nonlinear. The presence of delays in the control system is a phenomenon that can be seen as the dead-time between transmission and execution of an action. In this context, the delay is due to the fact that forces affecting the dynamics are associated with past events.

Time delays can be found in networked and distributed systems (Murray, 2003), communication networks (Kelly, 2001), teleoperation (Anderson and Spong, 1989), telesurgery (Speich and Rose, 2004), unmanned aerial vehicles (Ramírez et al., 2014), and decentralized control multiple collaborative agents (Beard et al., 2006), synchronization and haptics (Cheong et al., 2007), chemical processes (Marquez Rubio et al., 2012), population dynamics (Gopalsamy, 1992), biological phenomena (Niculescu et al., 2007), etc. Thus, the interest in understanding the effects of delays and in designing

* Corresponding author stabilizing controllers that take those delays into account has been increasing. Correspondingly, controllers in the time-delay context have become more and more complex.

The stability analysis of time-delay systems has two main approaches: in the frequency domain and in the time domain. In the former, necessary and sufficient conditions of stability are given (see, e.g., Bellman and Cooke, 1963; El'sgol'ts, 1966). The first and main results on stability of time delay systems were given by Kabakov (1946), Tzypkin (1946) and Neimark (1973). The Nyquist and Michailov criteria are the most commonly used results (Hahn, 1967; La Salle and Lefschetz, 1961). As for the time-domain approach, we have found two variants: the Lyapunov-Razumikhin approach (Razumikhin, 1956), which is an adaptation of the classical Lyapunov theory, and the Lyapunov-Krasovskii one (Krasovskii, 1956), which is a natural extension of Lyapunov theory to enable the use of Lyapunov functionals (Duda, 2012). Further developments for observer design of time-delay systems can be also found (Thuan et al., 2012).

On the other hand, design of nonlinear control systems has benefited from convex structures since the seminal work of Taniguchi et al. (2001), which showed a methodology to obtain an exact convex representation 
of a nonlinear model in a compact set of the state space. It has been proved that this model shares the properties of the well-known Takagi-Sugeno (TS) model (Takagi and Sugeno, 1985) and can therefore be analyzed using the direct Lyapunov method (Tanaka and Sugeno, 1990); conditions thus obtained are usually expressed as linear matrix inequalities (LMIs) which can be efficiently solved via convex optimization techniques already implemented in commercially available software (Boyd et al., 1994). A common structure for controller design under this approach has been the parallel distributed compensation (PDC), which employs the same convex functions of the model as to create a convex nonlinear controller that generalizes the ordinary state-feedback scheme (Wang et al., 1996; Tanaka and Wang, 2001).

As expected, time-delay systems have been studied via convex structures, too; this has been done in order to take advantage of the systematic design procedures which guarantee computational tractability via LMIs (Lin et al., 1991). In the TS context, the last decade has witnessed an abundance of results on a variety of issues related to time-delay systems: stability using the Lyapunov-Razumikhin functional approach (Cao and Frank, 2001), stability criteria for a class of nonlinear stochastic systems with time delay (Wang et al., 2004; Li et al., 2011; Balasubramaniam et al., 2012), design of feedback controllers design for nonlinear interval time-delay systems via a $\mathrm{T}-\mathrm{S}$ model (Chang et al., 2004), design of guaranteed cost control for T-S systems with delays (Chen and Liu, 2005; Chen et al., 2007b; 2007a; Kang and Wang, 2010) observer-based H-infinity control for time-delay T-S (Lin et al., 2007), $\alpha$-dissipative control for T-S fuzzy descriptor systems with time delay (Gassaraa et al., 2014). Most of these results take advantage of the possibility of rewriting Lyapunov-Krasovskii functionals as convex expressions by inheritance of the model convex structure: this investigation follows that path. In contrast to ordinary "fuzzy" approaches like that of Liu et al. (2014), this work does not consider approximations but convex exact representations of nonlinear models.

Based on a novel form of the Lyapunov-Krasovskii functional, this paper provides conditions for exponential estimates (a decay rate) of a class of time-delay nonlinear systems as well as guaranteed control cost of the control input. This is a topic that has been addressed very recently in several works (Zhang et al., 2009; Gassara et al., 2010; Chiu and Chiang, 2011); in contrast to those, this report has the following features: (a) a matrix relaxation based on Finsler's lemma which allows a natural inclusion of slack variables; (b) an exact convex representation of the plant which makes the proposed results directly valid for the original nonlinear setup; (c) guaranteed control cost as well as exponential bounds in a single framework, specifically designed for delayed inputs. The results are organized as follows: Section 2 introduces the definitions and notation this work is based on; Section 3 presents the main contributions in two theorems: the first one considers stability under a given delayed PDC control law, while the second provides controller synthesis for stabilization with guaranteed control cost and exponential estimates; Section 4 illustrates the effectiveness of the proposed scheme via a simulation example as well as a real-time implementation on the well-known twin rotor MIMO system, whose fast dynamics are particularly sensitive to time delays on the control input; and Section 5 gathers some concluding remarks and suggestions for future work.

\section{Preliminaries}

Consider an affine-in-control nonlinear system whose input is delayed in time:

$$
\begin{aligned}
\dot{x}(t) & =f(x(t)) x(t)+g(x(t)) u(t-\tau), \\
\phi(\theta) & =x(\theta), \quad \theta \in[-\tau, 0],
\end{aligned}
$$

where $f(\cdot): \mathbb{R}^{n} \rightarrow \mathbb{R}^{n \times n}$ is a matrix function such that $f(x(t)) x(t)$ is a Lipschitz vector field, $g(\cdot): \mathbb{R}^{n} \rightarrow$ $\mathbb{R}^{n \times m}$ is a matrix function, $u(t) \in \mathbb{R}^{m}$ is the input vector, $\tau \in \mathbb{R}^{+}$is a time delay, $\phi \in \mathfrak{C}\left([-\tau, 0], \mathbb{R}^{n}\right)$ is the initial function, and $\mathfrak{C}\left([-\tau, 0], \mathbb{R}^{n}\right)$ is the Banach space of real continuous functions on the interval $[-\tau, 0]$ with the following norm:

$$
\|\phi\|_{\tau}:=\max _{\theta \in[-\tau, 0]}\|\phi(\theta)\|,
$$

with $\|\cdot\|$ being the Euclidean norm in $\mathbb{R}^{n}$. It is assumed that for each initial condition $\phi \in \mathfrak{C}\left([-\tau, 0], \mathbb{R}^{n}\right)$ and $t \geq 0$ there exists a unique solution $x(t ; \phi)$ of the system. Here, $x_{t}(\phi):=\{x(t+\theta ; \phi): \theta \in[-\tau, 0]\} \in \mathbb{R}^{n}$ denote a segment of solution $x(t ; \phi)$, and it is the state vector which is assumed to lie in a compact set $\mathcal{C}$. When it does not cause an ambiguity, we write $x(t)$ and $x_{t}$ instead of $x(t ; \phi)$ and $x_{t}(\phi)$.

Results in this report are based on a convex rewriting of the nonlinear model (1). To this end, we will employ the sector nonlinearity methodology described by Taniguchi et al. (2001). If $f(\cdot)$ and $g(\cdot)$ have $p$ nonlinearities, they can be grouped in a vector

$$
z(\cdot)=\left[\begin{array}{llll}
z_{1}(\cdot) & z_{2}(\cdot) & \ldots & z_{p}(\cdot)
\end{array}\right]^{T},
$$

where each entry corresponds to a nonlinearity

$$
\begin{gathered}
z_{j}(\cdot) \in\left[\underline{z}_{j}, \bar{z}_{j}\right], \\
\underline{z}_{j}=\min _{x(t) \in \mathcal{C}} z_{j}(\cdot), \quad \bar{z}_{j}=\max _{x(t) \in \mathcal{C}} z_{j}(\cdot),
\end{gathered}
$$

$j \in\{1,2, \ldots, p\}$. The following weights are thus constructed:

$$
w_{0}^{j}(\cdot)=\frac{\bar{z}_{j}-z_{j}(\cdot)}{\bar{z}_{j}-\underline{z}_{j}}, \quad w_{1}^{j}(\cdot)=1-w_{0}^{j}(\cdot),
$$


with $j \in\{1,2, \ldots, p\}$. We can thus define the following functions:

$$
h_{i}=h_{1+i_{1}+i_{2} \times 2+\ldots+i_{p} \times 2^{p-1}}=\prod_{j=1}^{p} w_{i_{j}}^{j}\left(z_{j}\right),
$$

with $i \in\{1,2, \ldots, r\}, r=2^{p}, i_{j} \in\{0,1\}$, which hold the convex-sum property $\sum_{i=1}^{r} h_{i}(\cdot)=1, h_{i}(\cdot) \geq 0$, in $\mathcal{C}$.

Then, the nonlinear model (1) is rewritten in the following convex form:

$$
\begin{aligned}
& \dot{x}(t)=\sum_{i=1}^{r} h_{i}(z(t))\left(A_{i} x(t)+B_{i} u(t-\tau)\right), \\
& \phi(\theta)=x(\theta), \quad \theta \in[-\tau, 0],
\end{aligned}
$$

with $\phi(\theta)$ defined as before, $A_{i}=\left.f(z(t))\right|_{h_{i}=1}, B_{i}=$ $\left.g(z(t))\right|_{h_{i}=1}, i \in\{1,2, \ldots, r\}$. Sector nonlinearity guarantees that (4) is an exact representation of the nonlinear system described in (1) in $\mathcal{C}$; it is not an approximation.

Some of the expressions in the sequel that involve convex sums will be written shortly as

$$
\begin{gathered}
\Upsilon_{z}=\sum_{i=1}^{r} h_{i}(z(t)) \Upsilon_{i}, \\
\Upsilon_{z z}=\sum_{i=1}^{r} \sum_{j=1}^{r} h_{i}(z(t)) h_{j}(z(t)) \Upsilon_{i j}, \\
\Upsilon_{z, \tau}=\sum_{i=1}^{r} h_{i}(z(t-\tau)) \Upsilon_{i}, \\
\Upsilon_{z z}^{z, \tau}=\sum_{i=1}^{r} \sum_{j=1}^{r} \sum_{k=1}^{r} h_{i}(z(t)) h_{j}(z(t)) h_{k}(z(t-\tau)) \Upsilon_{i j}^{k}
\end{gathered}
$$

Following this notation, the convex model (4) can be written as $\dot{x}(t)=A_{z} x(t)+B_{z} u(t-\tau)$.

In order to obtain LMI conditions, MFs should be removed from nested convex sums. The following result will be employed to perform this task in triple convex sums, one of which will be delayed: it is an immediate extension of the relaxation lemma of Tuan et al. (2001):

Lemma 1. (Relaxation lemma) (Tuan et al., 2001) Let $\Upsilon_{i j}^{k}$ be matrices of proper dimensions. Then $\Upsilon_{z z}^{z, \tau}<0$ is implied by

$$
\begin{array}{r}
\Upsilon_{i i}^{k}<0, \quad \forall(i, k) \in\{1,2, \ldots, r\}^{2}, \\
\frac{2}{r-1} \Upsilon_{i i}^{k}+\Upsilon_{i j}^{k}+\Upsilon_{j i}^{k}<0, \\
\forall(i, j, k) \in\{1,2, \ldots, r\}^{3}, i \neq j .
\end{array}
$$

The following control law corresponds to the well-known parallel distributed compensation (PDC) (Wang et al., 1996) and will be employed in the sequel:

$$
u(t)=\sum_{j=1}^{r} h_{i}(z(t)) K_{j} x(t)=K_{z} x(t),
$$

with $K_{i} \in \mathbb{R}^{m \times n}, i \in\{1,2, \ldots, r\}$, being controller gains to be determined later. It is important to stress the fact that this control law is nonlinear because functions $h_{i}(\cdot)$ may depend nonlinearly on the states.

Substituting (6) in (4), the following closed-loop system equation is obtained:

$$
\begin{aligned}
\dot{x}(t) & =A_{z} x(t)+B_{z} K_{z, \tau} x(t-\tau), \\
\phi(\theta) & =x(\theta), \quad \theta \in[-\tau, 0],
\end{aligned}
$$

where $K_{z, \tau}=\sum_{j=1}^{r} h_{j}(z(t-\tau)) K_{j}$.

For brevity, symmetric terms in matrix expressions as well as in-line expressions will be denoted with a star $(*)$. Should a matrix expression be involved with symbols " $>$ " and " $<$ ", they will stand for positive and negative-definiteness, respectively. Arguments will be omitted when convenient.

The following matrix property will play a central role in obtaining the LMI conditions in this work.

Lemma 2. (Finsler's lemma) (Oliveira and Skelton, 2001) Let $x \in \mathbb{R}^{n}, Q=Q^{T} \in \mathbb{R}^{n \times n}$, and $R \in \mathbb{R}^{m \times n}$ such that $\operatorname{rank}(R)<n$. The following expressions are equivalent:

$$
\begin{aligned}
& \text { 1. } x^{T} Q x<0, \forall x \in\left\{x \in \mathbb{R}^{n}: x \neq 0, \Gamma x=0\right\} \text {, } \\
& \text { 2. } \exists \Omega \in \mathbb{R}^{n \times m}: Q+\Omega \Gamma+\Gamma^{T} \Omega^{T}<0 .
\end{aligned}
$$

We now turn our attention to the definition of guaranteed control cost and exponential estimates. For the first one, let $W \in \mathbb{R}^{n \times n}, W=W^{T}>0$ and $S \in \mathbb{R}^{m \times m}$, $S=S^{T}>0$; then, the following cost function will be used in the sequel (Chen et al., 2007a):

$$
J=\int_{0}^{\infty}\left(x^{T}(t) W x(t)+u^{T}(t-\tau) S u(t-\tau)\right) \mathrm{d} t .
$$

Guaranteed control cost control law. ( $Y u$ and $C h u$, 1999) For a nonlinear system of the form (1) (or, equivalently, (4)), if there exists a control input $u(t)$, a constant $J_{0}$ such that the closed-loop system is asymptotically stable, and $J \leq J_{0}$, where $J$ is given by (8), then $J_{0}$ is said to be a guaranteed cost and the control input $u(t)$ is called a guaranteed control cost control law for the system (1) (or, equivalently, (4)).

As for exponential estimates, the following result relating them with a Lyapunov-Krasovskii functional will be used.

Theorem 1. (Kharitonov and Hinrichsen, 2004) Consider the nonlinear system (1) or, equivalently, its convex TS representation (4). If there exist a functional $V(\cdot)$ and positive constants $\alpha_{1}, \alpha_{2}$, and $\sigma$ such that

$$
\begin{aligned}
& \text { 1. } \alpha_{1}\|x(t)\|^{2} \leq V\left(x_{t}\right) \leq \alpha_{2}\left\|x_{t}\right\|_{\tau}^{2}, \\
& \text { 2. } \dot{V}\left(x_{t}\right)+2 \sigma V\left(x_{t}\right)<0
\end{aligned}
$$


then the solutions $x(t ; \phi)$ of the system (1) (or, equivalently, (4)) satisfy the exponential estimates

$$
\|x(t ; \phi)\| \leq \sqrt{\frac{\alpha_{2}}{\alpha_{1}}} e^{-\sigma t}\|\phi\|_{\tau} .
$$

\section{Main results}

3.1. Stability. We begin by considering the stability analysis of the closed-loop time-delay system (7), which means that gains $K_{j}, j \in\{1,2, \ldots, r\}$ are already known. When stability analysis is performed, it is customary to provide these gains such that terms $A_{i}+$ $B_{i} K_{i}, j \in\{1,2, \ldots, r\}$ are Hurwitz; of course, this does not guarantee the stability of the whole scheme, which is why the stability analysis comes at hand. The next theorem should be therefore considered a first step towards the controller synthesis methodology shown in the next subsection.

Theorem 2. The closed-loop nonlinear system (7) with a time-delay $\mathbb{R} \ni \tau>0$ is exponentially stable with guaranteed cost

$$
\begin{aligned}
J_{0}= & x^{T}(0) P x(0)+\int_{-\tau}^{0} x^{T}(s) Q e^{2 \sigma s} x(s) \mathrm{d} s \\
& +\tau \int_{-\tau}^{0} \int_{\theta}^{0} \dot{x}^{T}(s) R e^{2 \sigma s} \dot{x}(s) \mathrm{d} s \mathrm{~d} \theta
\end{aligned}
$$

for the cost function (8) with $Q, P, R \in \mathbb{R}^{n \times n}$ such that $Q=Q^{T}>0, P=P^{T}>0, R=R^{T}>0$, and holds exponential estimates

$$
\|x(t ; \phi)\| \leq \sqrt{\frac{\alpha_{2}}{\alpha_{1}}} e^{-\sigma t}\|\phi\|_{\tau},
$$

for a decay rate $\mathbb{R} \ni \sigma>0$ with $\alpha_{1}=\lambda_{\min }(P), \alpha_{2}=$ $\lambda_{\max }(P)+\lambda_{\max }(Q) \tau+\lambda_{\max }(R) \tau$, if there exist matrices $W \in \mathbb{R}^{n \times n}: W=W^{T}>0, S \in \mathbb{R}^{m \times m}: S=S^{T}>0$, $K_{j} \in \mathbb{R}^{m \times n}, H_{i}^{p}, N_{i j}^{p} \in \mathbb{R}^{n \times n}, i, j \in\{1,2, \ldots, r\}$, $p \in\{1,2,3\}$ satisfying LMIs [5] for

$$
\Upsilon_{i j}^{k}=\left[\begin{array}{ccccc}
\psi_{11} & \psi_{12} & \psi_{13} & \psi_{14} & 0 \\
(*) & \psi_{22} & \psi_{23} & \psi_{24} & K_{k}^{T} \\
(*) & (*) & \psi_{33} & \psi_{34} & 0 \\
(*) & (*) & (*) & \psi_{44} & 0 \\
0 & (*) & 0 & 0 & -S^{-1}
\end{array}\right]
$$

where $i, j, k \in\{1,2, \ldots, r\}$, and

$$
\begin{aligned}
& \psi_{11}=H_{j}^{1} A_{i}+N_{i j}^{1}+(*)+2 P \sigma+Q+W, \\
& \psi_{12}=H_{j}^{1} B_{i} K_{k}+A_{i}^{T}\left(H_{j}^{2}\right)^{T}-N_{i j}^{1}+\left(N_{i j}^{2}\right)^{T}, \\
& \psi_{13}=-H_{j}^{1}+A_{i}^{T}\left(H_{j}^{3}\right)^{T}+\left(N_{i j}^{3}\right)^{T}+P, \\
& \psi_{14}=N_{i j}^{1}, \\
& \psi_{22}=H_{j}^{2} B_{i} K_{k}-N_{i j}^{2}+(*)-Q e^{2 \sigma \tau}, \\
& \psi_{23}=-H_{j}^{2}+K_{k}^{T} B_{i}^{T}\left(H_{j}^{3}\right)^{T}-\left(N_{i j}^{3}\right)^{T}, \\
& \psi_{24}=N_{i j}^{2}, \\
& \psi_{33}=\tau^{2} R-H_{j}^{3}-\left(H_{j}^{3}\right)^{T}, \\
& \psi_{34}=N_{i j}^{3}, \\
& \psi_{44}=-R e^{-2 \sigma \tau} .
\end{aligned}
$$

Proof. Consider the Lyapunov-Krasovskii functional of the form (Mondie and Kharitonov, 2005)

$$
\begin{aligned}
V\left(x_{t}\right)= & x^{T}(t) P x(t) \\
& +\int_{t-\tau}^{t} x^{T}(s) Q e^{2 \sigma(s-t)} x(s) \mathrm{d} s \\
& +\tau \int_{-\tau}^{0} \int_{t+\theta}^{t} \dot{x}^{T}(s) R e^{2 \sigma(s-t)} \dot{x}(s) \mathrm{d} s \mathrm{~d} \theta .
\end{aligned}
$$

Clearly, the above functional satisfies the following quadratics bounds:

$$
\alpha_{1}\|x(t)\|^{2} \leq V\left(x_{t}\right) \leq \alpha_{2}\left\|x_{t}\right\|_{\tau}^{2},
$$

where $\alpha_{1}=\lambda_{\min }(P)$ and $\alpha_{2}=\lambda_{\max }(P)+\lambda_{\max }(Q) \tau+$ $\lambda_{\max }(R) \tau$. We now turn our attention to the time derivative of 12):

$$
\begin{aligned}
\dot{V}\left(x_{t}\right) & \\
= & 2 x^{T}(t) P \dot{x}(t)+x^{T}(t) Q x(t) \\
& -x^{T}(t-\tau) Q e^{-2 \sigma \tau} x(t-\tau) \\
& -2 \sigma \int_{t-\tau}^{t} x^{T}(s) Q e^{2 \sigma(s-t)} x(s) \mathrm{d} s \\
& +\tau^{2} \dot{x}^{T}(t) R \dot{x}(t) \\
& -\tau \int_{t-\tau}^{t} \dot{x}^{T}(s) R e^{2 \sigma(s-t)} \dot{x}(s) \mathrm{d} s \\
& -2 \sigma \tau \int_{-\tau}^{0} \int_{t+\theta}^{t} \dot{x}^{T}(s) R e^{2 \sigma(s-t)} \dot{x}(s) \mathrm{d} s \mathrm{~d} \theta .
\end{aligned}
$$

Condition 2 in Theorem 1 translates into

$$
\begin{aligned}
\dot{V}+2 & \sigma V \\
= & 2 x^{T}(t) P \dot{x}(t)+2 \sigma x^{T}(t) P x(t) \\
& +x^{T}(t) Q x(t)-x^{T}(t-\tau) Q e^{-2 \sigma \tau} x(t-\tau) \\
& +\tau^{2} \dot{x}^{T}(t) R \dot{x}(t)
\end{aligned}
$$




$$
\begin{aligned}
& -\tau \int_{t-\tau}^{t} \dot{x}^{T}(s) R e^{2 \sigma(s-t)} \dot{x}(s) d s \\
= & \bar{x}^{T}\left[\begin{array}{ccc}
2 \sigma P+Q & 0 & P \\
0 & -Q e^{-2 \sigma \tau} & 0 \\
P & 0 & \tau^{2} R
\end{array}\right] \bar{x} \\
& -\tau \int_{t-\tau}^{t} \dot{x}^{T}(s) R e^{2 \sigma(s-t)} \dot{x}(s) \mathrm{d} s<0,
\end{aligned}
$$

where $\bar{x}=\left[\begin{array}{lll}x^{T}(t) & x^{T}(t-\tau) & \dot{x}^{T}(t)\end{array}\right]^{T}$. In this context, the convex model (7) can be rewritten as

$$
\left[\begin{array}{lll}
A_{z} & B_{z} K_{z, \tau} & -I
\end{array}\right]\left[\begin{array}{c}
x(t) \\
x(t-\tau) \\
\dot{x}(t)
\end{array}\right]=0
$$

whereas the fundamental theorem of calculus can be put in a similar fashion as follows:

$$
\left[\begin{array}{lll}
I & -I & 0
\end{array}\right]\left[\begin{array}{c}
x(t) \\
x(t-\tau) \\
\dot{x}(t)
\end{array}\right]-\int_{t-\tau}^{t} \dot{x}(s) \mathrm{d} s=0 .
$$

Finsler's lemma will be applied twice to combine the inequality (14) and the restrictions (15) and (16); it is important to notice that the integral terms in (14) and (16) will be included in Finsler's equivalence without loss of generality. Thus, after applying Finsler's lemma with the inequality (14) and the restriction (15), we have

$$
\begin{aligned}
\bar{x}^{T} & \left(\left[\begin{array}{c}
H_{z}^{1} \\
H_{z}^{2} \\
H_{z}^{3}
\end{array}\right]\left[\begin{array}{lll}
A_{z} & B_{z} K_{z, \tau} & -I
\end{array}\right]+(*)\right. \\
& \left.+\left[\begin{array}{ccc}
2 \sigma P+Q & 0 & P \\
0 & -Q e^{-2 \sigma \tau} & 0 \\
P & 0 & \tau^{2} R
\end{array}\right]\right) \bar{x} \\
& -\tau \int_{t-\tau}^{t} \dot{x}^{T}(s) R e^{2 \sigma(s-t)} \dot{x}(s) \mathrm{d} s \\
= & \bar{x}^{T} \Phi_{0} \bar{x} \\
& -\tau \int_{t-\tau}^{t} \dot{x}^{T}(s) R e^{2 \sigma(s-t)} \dot{x}(s) \mathrm{d} s<0,
\end{aligned}
$$

with $\Phi_{0}$ given in (18), $H_{z}^{p}, p \in\{1,2,3\}$ being single convex sums of matrices $H_{j}^{p} \in \mathbb{R}^{n \times n}, p \in\{1,2,3\}$, $j \in\{1, \ldots, r\}$. Notice that

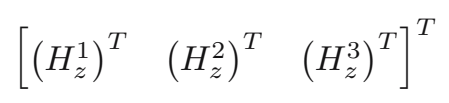

operates as $\Omega$ in Finsler's lemma (statement 1).

We now reapply Finsler's lemma with (17) as the new inequality and (16) as the new restriction. This yields

$$
\begin{aligned}
& \bar{x}^{T}\left(\left[\begin{array}{c}
N_{z z}^{1} \\
N_{z z}^{2} \\
N_{z z}^{3}
\end{array}\right]\left[\begin{array}{lll}
I & -I & 0
\end{array}\right]+(*)+\Phi_{0}\right) \bar{x} \\
& -\bar{x}^{T}\left[\begin{array}{c}
N_{z z}^{1} \\
N_{z z}^{2} \\
N_{z z}^{3}
\end{array}\right] \int_{t-\tau}^{t} \dot{x}(s) \mathrm{d} s-\int_{t-\tau}^{t} \dot{x}(s) \mathrm{d} s\left[\begin{array}{c}
N_{z z}^{1} \\
N_{z z}^{2} \\
N_{z z}^{3}
\end{array}\right]^{T} \bar{x} \\
& -\tau \int_{t-\tau}^{t} \dot{x}^{T}(s) R e^{2 \sigma(s-t)} \dot{x}(s) \mathrm{d} s \\
& =\bar{x}^{T}\left(\left[\begin{array}{ccc}
N_{z z}^{1}+\left(N_{z z}^{1}\right)^{T} & \left(N_{z z}^{2}\right)^{T}-N_{z z}^{1} & \left(N_{z z}^{3}\right)^{T} \\
N_{z z}^{2}-\left(N_{z z}^{1}\right)^{T} & -N_{z z}^{2}-\left(N_{z z}^{2}\right)^{T} & -\left(N_{z z}^{3}\right)^{T} \\
N_{z z}^{3} & -N_{z z}^{3} & 0
\end{array}\right]\right. \\
& \left.+\Phi_{0}\right) \bar{x}-2 \bar{x}^{T}\left[\begin{array}{c}
N_{z z}^{1} \\
N_{z z}^{2} \\
N_{z z}^{3}
\end{array}\right] \int_{t-\tau}^{t} \dot{x}(s) \mathrm{d} s \\
& -\tau \int_{t-\tau}^{t} \dot{x}^{T}(s) R e^{2 \sigma(s-t)} \dot{x}(s) \mathrm{d} s<0,
\end{aligned}
$$

with $N_{z z}^{p}, p \in\{1,2,3\}$ being double convex sums of matrices $N_{i j}^{p} \in \mathbb{R}^{n \times n}, p \in\{1,2,3\}, i, j \in\{1, \ldots, r\}$. Once again, notice that

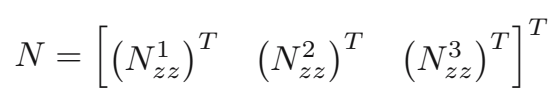

takes the place of $\Omega$ in Finsler's lemma (statement 1); notice also that the restriction in this case is extended from $\Gamma \bar{x}=0$ to the form $\Gamma_{1} \bar{x}+\Gamma_{2}=0$, where $\Gamma_{1}$ is the matrix term multiplied by $\bar{x}$ in (16) and $\Gamma_{2}$ is the integral term in the same expression. By the Park and Jensen inequalities (Gu et al., 2003), we have

$$
\begin{aligned}
-2 \bar{x}^{T}(t) N \int_{t-\tau}^{t} \dot{x}(s) \mathrm{d} s \\
\leq \bar{x}^{T}(t) N R^{-1} e^{2 \sigma \tau} N^{T} \bar{x}(t) \\
\quad+\int_{t-\tau}^{t} \dot{x}^{T}(s) \mathrm{d} s R e^{-2 \sigma \tau} \int_{t-\tau}^{t} \dot{x}(s) \mathrm{d} s \\
\leq \bar{x}^{T}(t) N R^{-1} e^{2 \sigma \tau} N^{T} \bar{x}(t) \\
\quad+\tau \int_{t-\tau}^{t} \dot{x}^{T}(s) R e^{-2 \sigma \tau} \dot{x}(s) \mathrm{d} s,
\end{aligned}
$$

which can be taken into account to cancel out the last integral term in 19 yielding

$$
\bar{x}^{T}\left(\Phi_{0}+\Phi_{1}+N R^{-1} e^{2 \sigma \tau} N^{T}\right) \bar{x}<0,
$$

with

$$
\Phi_{1}=\left[\begin{array}{ccc}
N_{z z}^{1}+\left(N_{z z}^{1}\right)^{T} & \left(N_{z z}^{2}\right)^{T}-N_{z z}^{1} & \left(N_{z z}^{3}\right)^{T} \\
N_{z z}^{2}-\left(N_{z z}^{1}\right)^{T} & -N_{z z}^{2}-\left(N_{z z}^{2}\right)^{T} & -\left(N_{z z}^{3}\right)^{T} \\
N_{z z}^{3} & -N_{z z}^{3} & 0
\end{array}\right] .
$$

The following development intends to include the guaranteed control cost in the previous analysis. To this 
end, consider matrices $\mathbb{R}^{n \times n} \ni W>0$ and $S \in \mathbb{R}^{m \times m}$ : $S>0$ from the integral argument in the cost function (8) by substituting (6):

$$
\begin{aligned}
& x^{T}(t) W x(t)+u^{T}(t-\tau) S u(t-\tau) \\
& \quad=x^{T}(t) W x(t)+x^{T}(t-\tau) K_{z, \tau}^{T} S K_{z, \tau} x(t-\tau) \\
& \quad=\bar{x}^{T}(t) \Phi_{2} \bar{x}(t),
\end{aligned}
$$

with

$$
\Phi_{2}=\left[\begin{array}{ccc}
W & 0 & 0 \\
0 & K_{z, \tau}^{T} S K_{z, \tau} & 0 \\
0 & 0 & 0
\end{array}\right]
$$

The inequality (21) guarantees that $\dot{V}+2 \sigma V<0$; keeping this in mind, as well as the equivalence in (22), it follows that

$$
\begin{aligned}
x^{T}(t) W x(t)+u^{T}(t-\tau) S u(t-\tau) \\
=x^{T}(t) W x(t)+u^{T}(t-\tau) S u(t-\tau)+\dot{V}-\dot{V} \\
<x^{T}(t) W x(t)+u^{T}(t-\tau) S u(t-\tau) \\
\quad+2 \sigma V+\dot{V}-\dot{V} \\
\quad<\bar{x}^{T}(t)\left(\Phi_{0}+\Phi_{1}+\Phi_{2}+N R^{-1} e^{2 \sigma \tau} N^{T}\right) \bar{x}(t) \\
\quad-\dot{V} .
\end{aligned}
$$

By the Schur complement we have that $\Phi_{0}+\Phi_{1}+$ $\Phi_{2}+N R^{-1} e^{2 \sigma \tau} N^{T}<0$ is equivalent to

$$
\left[\begin{array}{cc}
\Phi_{0}+\Phi_{1}+\Phi_{2} & N \\
N^{T} & -R e^{-2 \sigma \tau}
\end{array}\right]<0
$$

If $\left[\Phi_{0}\right]_{2,2}+\left[\Phi_{1}\right]_{2,2}+\left[\Phi_{2}\right]_{2,2}=\left[\Phi_{0}\right]_{2,2}+\left[\Phi_{1}\right]_{2,2}+$ $K_{z, \tau}^{T} S K_{z, \tau}$ denotes the block entry $(2,2)$ of the previous matrix, the Schur complement can be applied again yielding Eqn. (24). By the relaxation lemma, it is now clear that (24) holds if the LMIs (5) are satisfied for $\Upsilon_{i j}^{k}$, $i, j, k \in\{1,2, \ldots, r\}$ as defined in (11).

We can now return to the guaranteed cost function. Since $\dot{V}+2 \sigma V<0$ and bounds given on (13) have been established, Theorem 1 guarantees that solutions of the closed-loop model (7) satisfy (10). On the other hand, note that (24) guarantees $\Phi_{0}+\Phi_{1}+\Phi_{2}+N R^{-1} e^{2 \sigma \tau} N^{T}<$ 0 ; therefore, 23) is guaranteed if

$$
x^{T}(t) W x(t)+u^{T}(t-\tau) S u(t-\tau) \leq-\dot{V} .
$$

Integrating (25) on the interval $[0, T]$, we have

$$
\begin{aligned}
& \int_{0}^{T}\left(x^{T}(t) W x(t)+u^{T}(t-\tau) S u(t-\tau)\right) \mathrm{d} t \\
& \leq-V(T)+V(0) \\
& =-V(T)+x^{T}(0) P x(0)+\int_{-\tau}^{0} x^{T}(s) Q e^{2 \sigma s} x(s) \mathrm{d} s \\
& \quad+\tau \int_{-\tau}^{0} \int_{\theta}^{0} \dot{x}^{T}(s) R e^{2 \sigma s} \dot{x}(s) \mathrm{d} s \mathrm{~d} \theta .
\end{aligned}
$$

Since $\dot{V}<0, V(t)>0$ and $\exists V(T)>0$ as $T \rightarrow \infty$, we get

$$
\begin{aligned}
& \int_{0}^{\infty}\left(x^{T}(t) W x(t)+u^{T}(t-\tau) S u(t-\tau)\right) \mathrm{d} t \\
& \leq x^{T}(0) P x(0)+\int_{-\tau}^{0} x^{T}(s) Q e^{2 \sigma s} x(s) \mathrm{d} s \\
& \quad+\tau \int_{-\tau}^{0} \int_{\theta}^{0} \dot{x}^{T}(s) R e^{2 \sigma s} \dot{x}(s) \mathrm{d} s \mathrm{~d} \theta
\end{aligned}
$$

which means that the guaranteed cost (9) is satisfied, thus completing the proof.

Remark 1. Note that, traditionally, the constraints 15 and (16) are incorporated in the Lyapunov time-derivative (14) via zero additions instead of Finsler's lemma (Chen et al., 2007a). Nevertheless, both the approaches lead to the same results.

3.2. Stabilization. Theorem 1 assumes the gains $K_{i}$, $i \in\{1,2, \ldots, r\}$, are already given: it therefore performs a stability analysis. The next result provides sufficient conditions for stabilization (i.e., controller synthesis) of a nonlinear model of the form (1) under a delayed control law. As before, the result is obtained through a convex rewriting (4) of the original model (1), provided the control law (6) also shares the convex structure of this representation.

Theorem 3. The closed-loop nonlinear system (7) with time delay $\mathbb{R} \ni \tau>0$ is exponentially stable with guaranteed cost $J_{0}$ in (9) for the cost function (8) and holds the exponential estimates (10) for a decay rate $\mathbb{R} \ni \sigma>0$ with $\alpha_{1}=\lambda_{\min }(P), \alpha_{2}=\lambda_{\max }(P)+\lambda_{\max }(Q) \tau+$ $\lambda_{\max }(R) \tau$, if there exist matrices $\bar{Q}, \bar{P}, \bar{R}, W \in \mathbb{R}^{n \times n}$ such that $\bar{Q}=\bar{Q}^{T}>0, \bar{P}=\bar{P}^{T}>0, W=W^{T}>0$, $\mathbb{R}^{m \times m} \ni S=S^{T}>0, Y \in \mathbb{R}^{n \times n}, F_{k} \in \mathbb{R}^{m \times n}$,

$$
\Phi_{0}=\left[\begin{array}{ccc}
H_{z}^{1} A_{z}+(*)+2 \sigma P+Q & H_{z}^{1} B_{z} K_{z, \tau}+A_{z}^{T}\left(H_{z}^{2}\right)^{T} & P-H_{z}^{1}+A_{z}^{T}\left(H_{z}^{3}\right)^{T} \\
(*) & H_{z}^{2} B_{z} K_{z, \tau}+(*)-Q e^{-2 \sigma \tau} & -H_{z}^{2}+K_{z, \tau}^{T} B_{z}^{T}\left(H_{z}^{3}\right)^{T} \\
(*) & (*) & \tau^{2} R-H_{z}^{3}-\left(H_{z}^{3}\right)^{T}
\end{array}\right] .
$$




$$
\left.\Upsilon_{z z}^{z, \tau}=\left[\begin{array}{ccc}
{\left[\Phi_{0}+\Phi_{1}+\left[\begin{array}{ccc}
W & 0 & 0 \\
0 & 0 & 0 \\
0 & 0 & 0
\end{array}\right]\right.} & {\left[\begin{array}{c}
N_{z z}^{1} \\
N_{z z}^{2} \\
N_{z z}^{3}
\end{array}\right]} \\
(*) & & -R e^{-2 \sigma \tau}
\end{array}\right] \quad \begin{array}{c}
{\left[\begin{array}{c}
0 \\
K_{z, \tau}^{T} \\
0 \\
0
\end{array}\right]} \\
-S^{-1}
\end{array}\right]<0 .
$$

$k \in\{1,2, \ldots, r\}, \bar{N}_{p} \in \mathbb{R}^{n \times n}, p \in\{1,2,3\}$, and constants $a_{2}, a_{3} \in \mathbb{R}$, satisfying

$$
\left[\begin{array}{cccccc}
\overline{\psi_{11}} & \overline{\psi_{12}} & \overline{\psi_{13}} & \overline{\psi_{14}} & 0 & \overline{\psi_{16}} \\
(*) & \overline{\psi_{22}} & \overline{\psi_{23}} & \overline{\psi_{24}} & \overline{\psi_{25}} & 0 \\
(*) & (*) & \overline{\psi_{33}} & \overline{\psi_{3}} & 0 & 0 \\
(*) & (*) & (*) & \overline{\psi_{44}} & 0 & 0 \\
(*) & (*) & (*) & (*) & \overline{\psi_{55}} & 0 \\
(*) & (*) & (*) & (*) & (*) & \overline{\psi_{66}}
\end{array}\right]<0,
$$

where $i, k \in\{1,2, \ldots, r\}$, and

$$
\begin{aligned}
\overline{\psi_{11}}= & A_{i} Y^{T}+Y A_{i}^{T}+\bar{N}_{1}+{\overline{N_{1}}}^{T} \\
& +2 \sigma \bar{P}+\bar{Q}, \\
\overline{\psi_{12}}= & B_{i} F_{k}+a_{2} Y A_{i}^{T}-\bar{N}_{1}+{\overline{N_{2}}}^{T}, \\
\overline{\psi_{13}}= & -Y^{T}+a_{3} Y A_{i}^{T}+{\overline{N_{3}}}^{T}+\bar{P}, \\
\overline{\psi_{14}}= & \bar{N}_{1}, \\
\overline{\psi_{16}}= & Y \\
\overline{\psi_{22}}= & a_{2} B_{i} F_{k}+a_{2} F_{k}^{T} B_{i}^{T}-\bar{N}_{2} \\
& -\bar{N}_{2}^{T}-\bar{Q} e^{2 \sigma \tau}, \\
\overline{\psi_{23}}= & -a_{2} Y^{T}+a_{3} F_{k}^{T} B_{i}^{T}-\bar{N}_{3}^{T}, \\
\overline{\psi_{24}}= & \bar{N}_{2}, \\
\overline{\psi_{25}}= & F_{k}^{T} \\
\overline{\psi_{33}}= & -a_{3} Y^{T}-a_{3} Y+\tau^{2} \bar{R}, \\
\overline{\psi_{34}}= & \bar{N}_{3}, \\
\overline{\psi_{44}}= & -\bar{R} e^{-2 \sigma \tau}, \\
\overline{\psi_{55}}= & -S^{-1}, \\
\overline{\psi_{66}}= & -W^{-1} .
\end{aligned}
$$

The controller gains in (6) are thus calculated as $K_{k}=$ $F_{k} Y^{-T}, k \in\{1,2, \ldots, r\}$. The matrices corresponding to the Lyapunov-Krasovskii functional (12) are calculated as $P=Y^{-1} \bar{P} Y^{-T}, Q=Y^{-1} \bar{Q} Y^{-T}$ and $R=Y^{-1} \bar{R} Y^{-T}$.

Proof. Gains in (11) are multiplied by different sets of matrices $H_{j}^{p}$. In order to accomplish controller synthesis, these matrices are uniformized by choosing them as $H_{j}^{1}=$ $H_{1}, H_{j}^{2}=a_{2} H_{1}$, and $H_{j}^{3}=a_{3} H_{1}$, with $H_{1} \in \mathbb{R}^{n \times n}$ invertible and real constants $a_{2}, a_{3}$. This makes the same-instant crossed terms in (11) disappear; therefore, matrices $N_{i j}^{p}$ are also reduced as $N_{i j}^{1}=N_{1}, N_{i j}^{2}=N_{2}$, and $N_{i j}^{3}=N_{3}$, for $N_{1}, N_{2}, N_{3} \in \mathbb{R}^{n \times n}$.
Let $Y=H_{1}^{-1}$. The desired result easily arises after three steps:

1. Rewrite the expression (11) with the aforementioned reductions.

2. Pre- and post-multiply (11) by

$$
\text { block-diag }\left[\begin{array}{lllll}
Y & Y & Y & Y & I
\end{array}\right]
$$

and its transpose, respectively.

3. For the resulting expression, take the Schur complement of the block-entry $(1,1)$ over the term $Y W Y^{T}$.

Hence, the expression (26) arises, thus concluding the proof.

Remark 2. Notice that only indexes $i$ and $k$ remain in the expression, and that index $k$ corresponds to gains $K_{k}$ which occur at a different instant than indexes $i$ from the system, due to the time delay; therefore, no relaxation lemma can be applied here and all the combinations $(i, k)$ should be tested.

Remark 3. Theorem 3 is applicable to time-delay nonlinear systems exactly represented in the convex TS form via the sector nonlinearity approach (Taniguchi et al., 2001). This representation is not an approximation of the original model as happens with many other "fuzzy" methodologies such as that by Liu et al. (2014), which rely on fuzzy structures that are not fired simultaneously.

Remark 4. Convex structures in matrices $Q$ and $R$ of the Lyapunov-Krasovskii functional (12) can be easily exploited, since their time derivative will not include the time derivatives of the MFs $h_{i}(\cdot)$, i.e.,

$$
\begin{aligned}
& V\left(x_{t}\right) \\
& \quad=x^{T}(t) P x(t)+\int_{t-\tau}^{t} x^{T}(s) Q_{z} e^{2 \sigma(s-t)} x(s) \mathrm{d} s \\
& \quad+\tau \int_{-\tau}^{0} \int_{t+\theta}^{t} \dot{x}^{T}(s) R_{z} e^{2 \sigma(s-t)} \dot{x}(s) \mathrm{d} s \mathrm{~d} \theta,
\end{aligned}
$$

with $Q_{z}=\sum_{i=1}^{r} h_{i}(z) Q_{i}$ and $R_{z}=\sum_{i=1}^{r} h_{i}(z) R_{i}$. Clearly, this may provide additional degrees of freedom to relax the aforementioned results (Liu et al., 2014). 


\section{Implementation on the twin rotor MIMO system}

In order to illustrate the applicability of the proposed approach in a real-time application whose behavior is highly sensitive to time delays, this section considers the twin rotor MIMO system (TRMS) (Fee, 1998). Most of the state-space representations of the TRMS have 6 states (Nejjari et al., 2011; Pratap and Purwar, 2010; Tao et al., 2010): the elevation and azimuth angles, their corresponding angular speeds, and the motor speeds. Some other models add a coupling internal state; this is the case of the TRMS model employed in this investigation (Fee, 1998; Ahmed et al., 2009; Gonzalez et al., 2012). Thus, considering $x_{1}$ to be the main motor speed, $x_{2}$ the elevation angle, $x_{3}$ the elevation angular speed, $x_{4}$ the tail motor speed, $x_{5}$ the azimuth angle, $x_{6}$ the azimuth angular speed, and $x_{7}$ a coupling internal state, the TRMS model is given by (Fee, 1998; Gonzalez et al., 2012)

$$
\dot{x}(t)=f(x) x(t)+g(x) u(t),
$$

with $x(t)=\left[\begin{array}{lllllll}x_{1} & x_{2} & x_{3} & x_{4} & x_{5} & x_{6} & x_{7}\end{array}\right]^{T}$ being the state vector, $u(t) \in \mathbb{R}^{2 \times 1}$ being the input vector (recall the system has two actuators corresponding to the motors torque), and

$$
\begin{aligned}
f(x)= & {\left[\begin{array}{ccccccc}
-0.83 & 0 & 0 & 0 & 0 & 0 & 0 \\
0 & 0 & 1 & 0 & 0 & 0 & 0 \\
E_{1}(x) & E_{2}(x) & -\frac{0.006}{I_{1}} & 0 & 0 & E_{3}(x) & 0 \\
0 & 0 & 0 & -1 & 0 & 0 & 0 \\
0 & 0 & 0 & 0 & 0 & 1 & 0 \\
0 & 0 & 0 & E_{4}(x) & 0 & -\frac{0.1}{I_{2}} & -\frac{1}{I_{2}} \\
E_{5}(x) & 0 & 0 & 0 & 0 & 0 & -0.5
\end{array}\right] } \\
g(x)= & {\left[\begin{array}{cc}
0.917 & 0 \\
0 & 0 \\
0 & 0 \\
0 & 0.8 \\
0 & 0 \\
0 & 0 \\
E_{6}(x) & 0
\end{array}\right], }
\end{aligned}
$$

$$
\begin{aligned}
& E_{1}(x)=\left(b_{1}+a_{1} x_{1}\right)\left(\frac{1-0.05 x_{6} \cos x_{2}}{I_{1}}\right), \\
& E_{2}(x)=-\frac{0.32 \sin x_{2}}{I_{1} x_{2}}, \\
& E_{3}(x)=\frac{0.0163 x_{6} \sin \left(2 x_{2}\right)}{I_{1}}, \\
& E_{4}(x)=\frac{b_{2}+a_{2} x_{4}}{I_{2}}, \\
& E_{5}(x)=-0.1\left(b_{1}+a_{1} x_{1}\right)+0.583\left(\frac{b_{1}}{2}+a_{1} x_{1}\right), \\
& E_{6}(x)=\frac{1.1 A_{\text {cte }}}{1.2}\left(\frac{b_{1}}{2}+a_{1} x_{1}\right),
\end{aligned}
$$

$A_{\text {cte }}=-0.7, a_{1}=0.0135, a_{2}=0.02, b_{1}=0.0924$, $b_{2}=0.09, I_{1}=0.068$, and $I_{2}=0.02$.
An exact convex representation of the nonlinear model 29) can be found through the sector nonlinearity approach described in Section 2 by taking $z_{i} \in\left[\underline{z}_{i}, \bar{z}_{i}\right]$ as

$$
\begin{aligned}
& z_{1}=x_{1} \in[-0.8,0.8], \\
& z_{2}=x_{6} \cos x_{2} \in[-0.5574,0.8], \\
& z_{3}=\frac{\sin x_{2}}{x_{2}} \in[0.8967,1], \\
& z_{4}=x_{6} \sin \left(2 x_{2}\right) \in[-0.8,0.8], \\
& z_{5}=x_{5} \in[-0.6,0.6]
\end{aligned}
$$

from which weighing functions

$$
\begin{gathered}
\omega_{0}^{i}=\frac{\bar{z}_{i}-z_{i}}{\bar{z}_{i}-\underline{z}_{i}}, \\
\omega_{1}^{1}=1-\omega_{0}^{i}
\end{gathered}
$$

are defined to produce MFs

$$
h_{i}=h_{1+i_{1}+i_{2} \times 2+\ldots+i_{5} \times 2^{4}}=\prod_{j=1}^{5} w_{i_{j}}^{j}\left(z_{j}\right) .
$$

The resulting model has the form

$$
\dot{x}(t)=\sum_{i=1}^{32} h_{i}(z(t))\left(A_{i} x(t)+B_{i} u(t)\right),
$$

with $\left(A_{i}, B_{i}\right)=\left.(f(x), g(x))\right|_{h_{i}=1}$.

Conditions in Theorem 3 were found feasible in the MATLAB LMI Toolbox (Gahinet et al., 1995) for a time delay of $\tau=0.27$ and decay rate $\sigma=0.25$. The resulting PDC control law is (6) with 32 gains $K_{i}$, which, along with the MFs $h_{i}, i \in\{1,2, \ldots, 32\}$, constitute the convex sum $K_{z}$. Here, the exponential estimates for initial condition $\phi=\left[\begin{array}{lllllll}0 & 0.57 & 0 & 0 & -0.4 & 0 & 0\end{array}\right]$ are

$$
\begin{aligned}
\|x(t ; \phi)\| & \leq \sqrt{\frac{\alpha_{2}}{\alpha_{1}}} e^{-\sigma t}\|\phi\|_{\tau} \\
& =46.6971 e^{-0.25 t}\|\phi\|_{0.27},
\end{aligned}
$$

with a guaranteed cost of $J_{0}=16.4828$. The Lyapunov-Krasovskii functional matrices and some of the 32 gains are shown below.

The time evolution of the TRMS states under the time-delayed control law above is shown on the left in Fig. 1 and the control law is depicted on the right. It can be seen that, despite the time delay, stabilization as well as the exponential bound are achieved.

The TRMS employed for real time-implementation only provides states $x_{2}, x_{3}, x_{5}$, and $x_{6}$, which are measurements from the encoder sensors; the remaining states have to be estimated (Fee, 1998). As can be checked in the user's guide, states $x_{1}, x_{4}$, and $x_{7}$ can be filtered from the input torques; note that this task does not lead to algebraic loops if the input is delayed. Thus, real-time implementation of the simulation results above is shown in Fig. 2. The peaks presented in the figure are user-induced perturbations. 


\section{Conclusions}

A novel approach to stability and stabilization of nonlinear systems with delayed multivariable inputs providing exponential estimates as well as a guaranteed cost of the system solutions has been presented. An exact convex representation of the system in a compact set of the state-space has been used altogether with a Lyapunov-Krasovskii functional to obtain sufficient conditions in the form of linear matrix inequalities, which are efficiently solved via convex optimization techniques. In contrast to other results on the subject, this development makes use of recent techniques such as Finsler's lemma in order to combine inequalities and restrictions in a single unified framework. The developed approach has been successfully implemented in real time on the twin rotor MIMO system.

\section{Acknowledgment}

The authors gratefully acknowledge the following sponsors: project UAEH FPROMEP39/Rev-03, project ITSON CA-18 PROFAPI 00468 and project CONACYT CB-2011-168406, as well as CONACYT scholarships with CVU 489660 and CVU 18871.

\section{References}

Ahmed, Q., Bhatti, A. and Iqbal, S. (2009). Robust decoupling control design for twin rotor system using Hadamard weights, Control Applications, (CCA) Intelligent Control, (ISIC), 2009 IEEE, St. Petersburg, Russia, pp. 1009-1014.

Anderson, R.J. and Spong, M.W. (1989). Bilateral control of teleoperators with time delay, IEEE Transactions on Automatic Control 34(1): 494-501.

$$
\begin{gathered}
K_{1}=\left[\begin{array}{ccccccc}
-3.3417 & 8.1422 & -2.2709 & -0.0257 & -0.0234 & 0.0001 & 0.5179 \\
1.4659 & -2.8253 & 0.6360 & -2.0600 & -2.1259 & -0.7830 & 31.8786
\end{array}\right], \\
K_{9}=\left[\begin{array}{ccccccc}
-3.3136 & 8.0995 & -2.2526 & -0.0279 & -0.0268 & -0.0057 & 0.5569 \\
1.4599 & -2.7957 & 0.6340 & -2.0073 & -2.1000 & -0.7624 & 31.0891
\end{array}\right], \\
K_{17}=\left[\begin{array}{ccccccc}
-3.2913 & 8.0672 & -2.2397 & -0.0253 & -0.0277 & -0.0130 & 0.4945 \\
1.4596 & -2.7905 & 0.6354 & -2.0037 & -2.0985 & -0.7545 & 31.0708
\end{array}\right], \\
K_{25}=\left[\begin{array}{cccccccc}
-3.2730 & 8.0429 & -2.2310 & -0.0218 & -0.0285 & -0.0205 & 0.4122 \\
1.4660 & -2.8019 & 0.6441 & -2.0277 & -2.1108 & -0.7590 & 31.4627
\end{array}\right], \\
\left.\begin{array}{cccccccc}
8.1141 & -12.5152 & 10.1272 & -0.4417 & -0.5756 & -0.1370 & 12.3295 \\
-12.5152 & 46.3667 & -9.8052 & 0.5021 & 0.5912 & 0.2876 & -6.5328 \\
10.1272 & -9.8052 & 20.4008 & 0.1383 & 0.0581 & -0.0395 & -2.6478 \\
-0.4417 & 0.5021 & 0.1383 & 10.5371 & 5.0784 & -3.3166 & -119.3204 \\
-0.5756 & 0.5912 & 0.0581 & 5.0784 & 10.3226 & 1.6848 & -63.9367 \\
-0.1370 & 0.2876 & -0.0395 & -3.3166 & 1.6848 & 6.6901 & 35.6074 \\
12.3295 & -6.5328 & -2.6478 & -119.3204 & -63.9367 & 35.6074 & 1594.0950
\end{array}\right], \\
Q= \\
{\left[\begin{array}{cccccccc}
0.0039 & 0.0003 & 0.0037 & 0.0057 & 0.0067 & -0.0243 & 0.0001 \\
0.0003 & 0.0003 & -0.0011 & 0.0009 & 0.0021 & -0.0050 & 0.0000 \\
0.0037 & -0.0011 & 0.0124 & -0.0040 & -0.0031 & 0.0113 & -0.0001 \\
0.0057 & 0.0009 & -0.0040 & 0.2355 & 0.0055 & -0.2075 & 0.0185 \\
0.0067 & 0.0021 & -0.0031 & 0.0055 & 0.0803 & -0.0606 & 0.0023 \\
-0.0243 & -0.0050 & 0.0113 & -0.2075 & -0.0606 & 0.9222 & 0.0060 \\
0.0001 & 0.0000 & -0.0001 & 0.0185 & 0.0023 & 0.0060 & 0.0023
\end{array}\right],} \\
{\left[\begin{array}{cccccccc}
5.3827 & 0.2710 & -2.4988 & -0.5332 & -0.0164 & 0.0340 & -0.1191 \\
0.2710 & 0.2200 & -0.0152 & -0.0533 & -0.0029 & -0.0180 & -0.0025 \\
-2.4988 & -0.0152 & 1.4002 & 0.2484 & 0.0067 & -0.0283 & 0.0597 \\
-0.5332 & -0.0533 & 0.2484 & 3.7296 & -0.0178 & 0.1266 & 0.2588 \\
-0.0164 & -0.0029 & 0.0067 & -0.0178 & 1.9919 & -0.4440 & 0.0927 \\
0.0340 & -0.0180 & -0.0283 & 0.1266 & -0.4440 & 2.9497 & 0.0015 \\
-0.1191 & -0.0025 & 0.0597 & 0.2588 & 0.0927 & 0.0015 & 0.0540
\end{array}\right] .}
\end{gathered}
$$




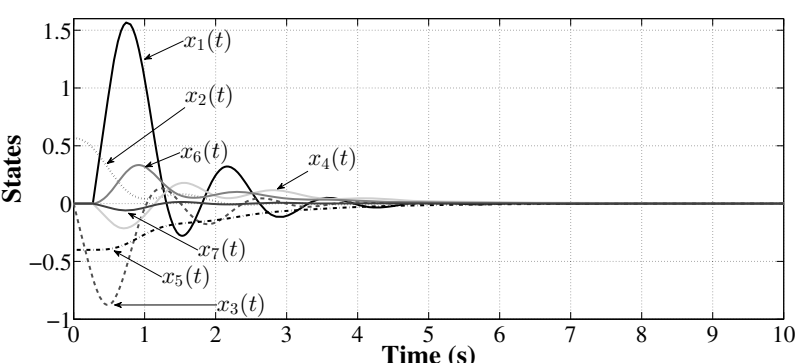

(a)

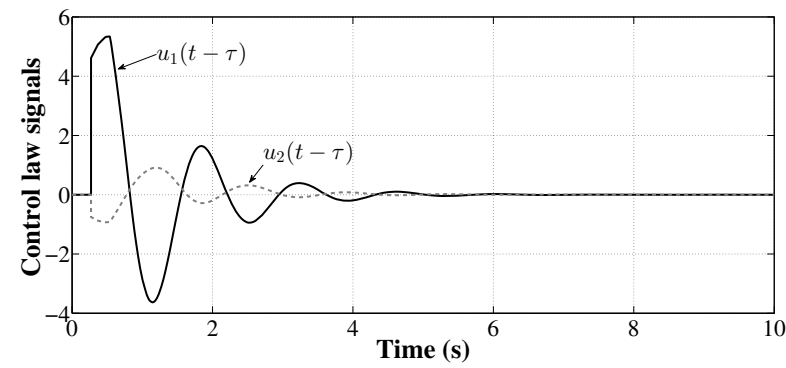

(b)

Fig. 1. State evolution of the TRMS under the control law (6) obtained via Theorem 3 with $\tau=0.27, \sigma=0.25$ (a). Time evolution of the control signals (b).

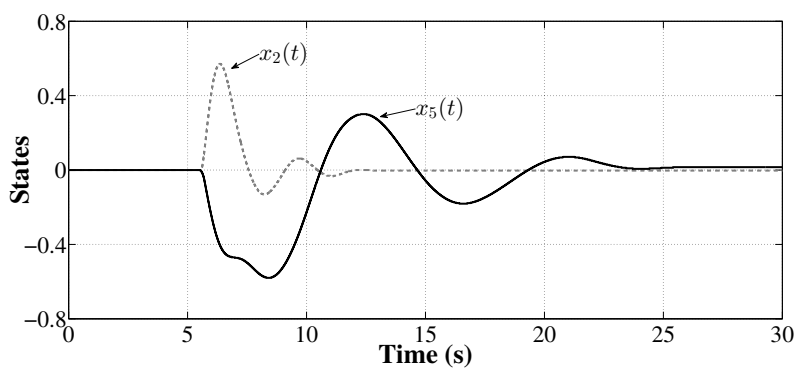

(a)

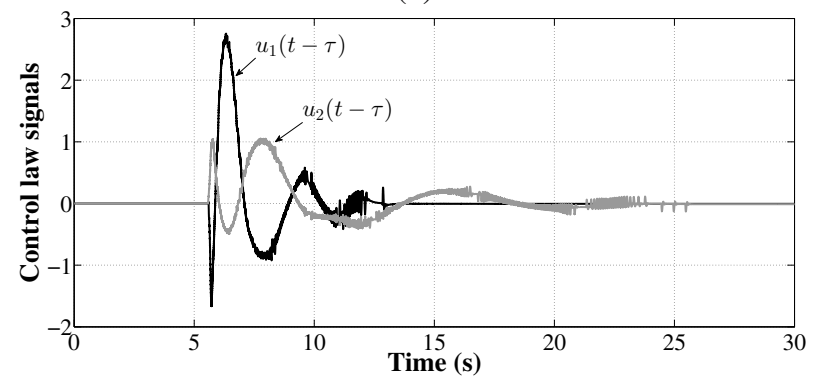

(b)

Fig. 2. State evolution of the TRMS under the control law (6) obtained via Theorem 3 with $\tau=0.27, \sigma=0.25$ (a). Time evolution of the control signals (b).

Balasubramaniam, P., Lakshmanan, S. and Rakkiyappan, R. (2012). LMI optimization problem of delay-dependent robust stability criteria for stochastic systems with polytopic and linear fractional uncertainties, International Journal of Applied Mathematics and Computer Science
22(2): 339-351, DOI: 10.2478/v10006-012-0025-6.

Beard, W.B., McLain, T.W., Nelson, D.B., Kingston, D. and Johanson, D. (2006). Decentralized cooperative aerial surveillance using fixed-wing miniature UAVs, Proceedings of the IEEE 94(1): 1306-1324.

Bellman, R. and Cooke, K. (1963). Differential-Difference Equations, Academic Press, New York, NY.

Boyd, S., Ghaoui, L.E., Feron, E. and Belakrishnan, V. (1994). Linear Matrix Inequalities in System and Control Theory, Vol. 15, SIAM, Philadelphia, PA.

Cao, Y.Y. and Frank, P.M. (2001). Stability analysis and synthesis of nonlinear time-delay systems via linear Takagi-Sugeno fuzzy models, Fuzzy Sets and Systems 124(2): 213-229.

Chang, Y.C., Chen, S., Su, S. and Lee, T. (2004). Static output feedback stabilization for nonlinear interval time-delay systems via fuzzy control approach, Fuzzy Sets and Systems 148(3): 395-410.

Chen, B., Lin, C., Liu, X. and Tong, S. (2007a). Guaranteed cost control of T-S fuzzy systems with input delay, International Journal of Robust Nonlinear Control 18(1): 1230-1256.

Chen, B., Liu, X.and Tong, S. and Lin, C. (2007b). Guaranteed cost control of T-S fuzzy systems with state and input delays, Fuzzy Sets and Systems 158(20): 2251-2267.

Chen, B. and Liu, X. (2005). Fuzzy guaranteed cost control for nonlinear systems with time-varying delay, IEEE Transactions on Fuzzy Systems 13(2): 238-249.

Cheong, Niculescu, S.-I., Annaswamy, A. and Srinivasan, A. (2007). Synchronization control for physics-based collaborative virtual environments with shared haptics, $A d$ vanced Robotics 21(1): 1001-1029.

Chiu, C.-S. and Chiang, T.-S. (2011). Observer-based exponential stabilization of Takagi-Sugeno fuzzy systems with state and input delays, Journal of Systems and Control Engineering 225(7): 993-1004.

Duda, J. (2012). A Lyapunov functional for a system with a time-varying delay, International Journal of Applied Mathematics and Computer Science 22(2): 327-337, DOI: 10.2478/v10006-012-0024-7.

El'sgol'ts, L.E. (1966). Introduction to the Theory of Differential Equations with Deviating Arguments, Holden-Day, San Francisco, CA.

Fee (1998). Twin Rotor MIMO System. Advanced Teaching Manual 1, 33-007-4M5.

Gahinet, P., Nemirovski, A., Laub, A.J. and Chilali, M. (1995). LMI Control Toolbox, MathWorks, Natick, MA.

Gassara, H., El-Hajjaji, A. and Chaabane, M. (2010). Delay-dependent $H$-infinite exponential stabilization of T-S fuzzy systems with interval time-varying delay, Proceeding of the 49th IEEE Conference on Decision and Control, Atlanta, GA, USA, pp. 4281-4286.

Gassaraa, A., El Hajjajia, A., Kchaoub, M. and Chaabaneb, M. (2014). Observer based (q,v,r)- $\alpha$-dissipative control for TS fuzzy descriptor systems with time delay, Journal of the Franklin Institute 351(1): 187-206. 
Gonzalez, T., Rivera, P. and Bernal, M. (2012). Nonlinear control for plants with partial information via Takagi-Sugeno models: An application on the twin rotor MIMO system, 2012 9th International Conference on Electrical Engineering, Computing Science and Automatic Control (CCE), DF, México, pp. 1-6.

Gopalsamy, K. (1992). Stability and Oscillations in Delay Differential Equations of Population Dynamics, Kluwer, Norwell, MA.

Gu, K., Kharotonov, V. and Chen, J. (2003). Stability of Time Delay Systems, Birkhauser, Basel.

Hahn, W. (1967). Stability of Motion, Springer-Verlag, Berlin.

Kabakov, I. (1946). Concerning the control process for the steam pleasure, Inzhenernii Sbornik 2(1): 27-76.

Kang, Q. and Wang, W. (2010). Guaranteed cost control for T-S fuzzy systems with time-varying delays, Journal of Control Theory and Applications 8(4): 413-417.

Kelly, F.P. (2001). Mathematical modelling of the internet, in B. Engquist and W. Schmid (Eds.), Mathematics Unlimited-2001 and Beyond, Vol. 1, Springer-Verlag, Berlin, pp. 685-702.

Kharitonov, V. and Hinrichsen, D. (2004). Exponential estimates for time delay systems, Systems \& Control Letters 53(1): 395-405.

Krasovskii, N. (1956). On the application of the second method of Lyapunov for equations with time delays, Prikladnaya Matematika i Mekhanika 20(3): 315-327.

La Salle, J. and Lefschetz, S. (1961). Stability by Lyapunov's Direct Method: With Applications, Academic Press, London.

Li, J., Li, J. and Xia, Z. (2011). Delay-dependent generalized $\mathrm{H}_{2}$ control for discrete T-S fuzzy large-scale stochastic systems with mixed delays, International Journal of Applied Mathematics and Computer Science 21(4): 585-603, DOI: 10.2478/v10006-011-0046-6.

Lin, C., Wang, Q., Lee, T.H. and Chen, B. (2007). Observer-based $h_{\infty}$ control for T-S fuzzy systems with time delay: Delay-dependent design method, IEEE Transactions on Systems, Man, and Cybernetics, Part B: Cybernetics 34(4): 1030-1038.

Lin, C., Wang, Q., Lee, T.H. and He, Y. (1991). LMI Approach to Analysis and Control of Takagi-Sugeno Fuzzy Systems with Time-Delay, Prentice Hall, New York, NY.

Liu, H., Shi, P., Karimi, H. and Chadli, M. (2014). Finite-time stability and stabilisation for a class of nonlinear systems with time-varying delay, International Journal of Systems Science 1(1): 1-12.

Marquez Rubio, J.F., del Muro Cuéllar, B. and Sename, O. (2012). Control of delayed recycling systems with an unstable pole at forward path, American Control Conference (ACC), Montreal, Canada, pp. 5658-5663.

Mondie, S. and Kharitonov, V. (2005). Exponential estimates for retarded time-delay systems: An LMI approach, IEEE Transactions on Automatic Control 50(2): 268-273.

Murray, R.M. (Ed.) (2003). Control in an Information Rich World: Report of the Panel on Future Directions in Control, SIAM, Philadelphia, PA.
Neimark, J.I. (1973). D-decomposition of spaces of quasi-polynomials, American Mathematical Society Translations 102(2): 95-131.

Nejjari, F., Rotondo, D., Puig, V. and Innocenti, M. (2011). LPV modelling and control of a twin rotor MIMO system, 19th Mediterranean Conference on Control Automation (MED), 2011, Corfu, Greece, pp. 1082-1087.

Niculescu, S.-I., Morărescu, C., Michiels, W. and Gu, K. (2007). Geometric ideas in the stability analysis of delay models in biosciences, in I. Queinnec et al. (Eds.), Biology and Control Theory: Current Challenges, Lecture Notes in Control and Information Sciences, Vol. 317, Springer Verlag, Berlin/Heidelberg, pp. 217-259.

Oliveira, M. and Skelton, R. (2001). Stability tests for constrained linear systems, in S.Q.R. Moheimani (Ed.), Perspectives in Robust Control, Lecture Notes in Control and Information Sciences, Vol. 268, Springer-Verlag, Berlin, pp. 241-257.

Pratap, B. and Purwar, S. (2010). Neural network observer for twin rotor MIMO system: An LMI based approach, 2010 International Conference on Modelling, Identification and Control (ICMIC), Okayama, Japan, pp. 539-544.

Ramírez, A., Espinoza, E.S., García, L.R., Mondié, S., García, A. and Lozano, R. (2014). Stability analysis of a vision-based UAV controller, Journal of Intelligent and Robotic Systems 74(1): 69-84.

Razumikhin, B. (1956). On stability of systems with a delay, Prikladnaya Matematika i Mekhanika 20(1): 500-512.

Speich, E. and Rose, J. (2004). Medical Robotics, Prentice Hall, Marcel Dekker, New York, NY.

Takagi, T. and Sugeno, M. (1985). Fuzzy identification of systems and its application to modeling and control, IEEE Transactions on Systems Man and Cybernetics 15(1): 116-132.

Tanaka, K. and Sugeno, M. (1990). Stability analysis of fuzzy systems using Lyapunov's direct method, NAFIPS'90, Kanazawa, Japan, pp. 133-136.

Tanaka, K. and Wang, H. (2001). Fuzzy Control Systems Design and Analysis. A Linear Matrix Inequality Approach, John Wiley \& Sons, New York, NY.

Taniguchi, T., Tanaka, K. and Wang, H. (2001). Model construction, rule reduction and robust compensation for generalized form of Takagi-Sugeno fuzzy systems, IEEE Transactions on Fuzzy Systems 9(2): 525-537.

Tao, C., Taur, J.-S., Chang, Y.-H. and Chang, C.-W. (2010). A novel fuzzy-sliding and fuzzy-integral-sliding controller for the twin-rotor multi-input-multi-output system, IEEE Transactions on Fuzzy Systems 18(5): 893-905.

Thuan, M.V., Phat, V.N. and Trinh, H. (2012). Observer-based controller design of time-delay systems with an interval time-varying delay, International Journal of Applied Mathematics and Computer Science 22(4): 921-927, DOI: 10.2478/v10006-012-0068-8.

Tuan, H., Apkarian, P., Narikiyo, T. and Yamamoto, Y. (2001). Parameterized linear matrix inequality techniques in fuzzy control system design, IEEE Transactions on Fuzzy Systems 9(2): 324-332. 
Tzypkin, J. (1946). Stability of systems with delayed feedback, Automatic and Remote Control 7(2): 107-129.

Wang, H., Tanaka, K. and Griffin, M. (1996). An approach to fuzzy control of nonlinear systems: Stability and design issues, IEEE Transactions on Fuzzy Systems 4(1): 14-23.

Wang, Z., Ho, D. and Liu, X. (2004). A note on the robust stability of uncertain stochastic fuzzy systems with time-delays, IEEE Transactions on System, Man, and Cybernetics A 34(4): 570-576.

Yu, L. and Chu, J. (1999). An LMI approach to guaranteed cost control of linear uncertain time-delay systems, Automatica 35(1): 1155-1159.

Zhang, B., Lam, J., Xu, S. and Shu, Z. (2009). Robust stabilization of uncertain T-S fuzzy time-delay systems with exponential estimates, Fuzzy Sets and Systems 160(12): 1720-1737.

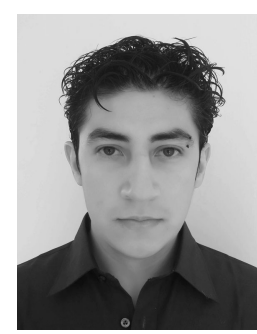

Máximo Ramírez Melo received the B.Sc. degree in mechatronic engineering from Universidad Politécnica de Pachuca, Mexico, in 2012, and the M.Sc. degree in automation and control sciences from Universidad Autónoma del Estado de Hidalgo, Mexico, in 2014. Currently, he is a Ph.D. student at the Automatic Control Department at CINVESTAV/Guadalajara Unit, Mexico. His research interest includes nonlinear control, time delay systems and neural networks design.

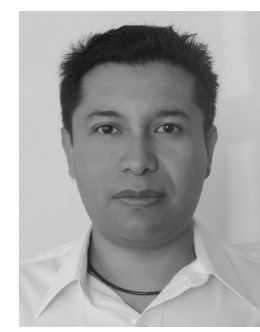

Raúl Villafuerte Segura received the B.Sc. degree in physics and mathematics engineering from ESFM-IPN, Mexico City, Mexico, in 2004, and the M.Sc. and the Ph.D. degrees in automatic control from CINVESTAV-IPN, Mexico City, in 2006 and 2010, respectively. Since 2011, he has been a professor at Hidalgo State University (UAEH), Mexico. He has been a member of the National Research System of Mexico since 2012. His research interests include time delay systems and nonlinear controller design.

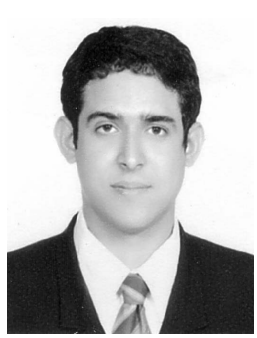

Iván Temoatzin González Germán received the B.Sc. and M.Sc. degrees in electrical engineering from Instituto Tecnológico de Sonora, Mexico, in 2012 and 2014, respectively. He currently works toward the Ph.D. degree at Universitat Politécnica de Valéncia. His present research interests include analysis and synthesis of nonlinear control systems through convex programming (i.e., linear matrix inequalities or sum of squares) for dynamic systems.

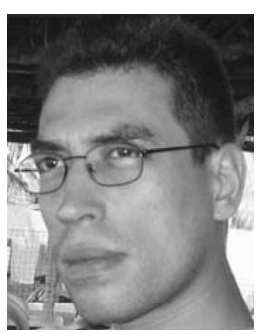

Miguel Ángel Bernal Reza received the Ph.D degree in automatic control from Czech Technical University, Prague, Czech Republic, in 2005. $\mathrm{He}$ is currently an associate professor with the Sonora Institute of Technology. He is a member of the National Research System of Mexico and has taught at several universities there. His current research interests include analysis and synthesis of nonlinear control systems through convex programming (i.e., linear matrix inequalities or sum of squares) for dynamical systems.

Received: 2 August 2014 Revised: 14 April 2015 